\title{
Black Holes in Models with Dilaton Field and Electric or Electric and Magnetic Charges
}

\author{
E. Kyriakopoulos 1 \\ Department of Physics \\ National Technical University \\ 15780 Zografou, Athens, GREECE
}

\begin{abstract}
Exact static spherically symmetric charged black holes in four dimensions are presented. One of them has only electric charge and another electric and magnetic charges. In these solutions the metric is asymptotically flat, has two horizons, irremovable singularity only at $r=0$, and the dilaton field is singular only at $r=0$. The solution with electric charge only is characterized by three free parameters, the ADM mass, the electric charge and an additional free parameter. It can be considered as a modification of the GHS-GM solution obtained by changing the coupling between dilaton and electromagnetic field. The general dyonic solution is again characterized by three free parameters, the ADM mass, the magnetic charge and an additional free parameter, which is not the electric charge. According to a definition of the no-hair conjecture the solutions are "hairy".A very interesting special case of the dyonic solution is characterized by three free parameters, the ADM mass and the electric and the magnetic charges. The solutions satisfy the dominant as well as the strong energy condition outside and on the external horizon.
\end{abstract}

PACS number(s): 04.20.Jb, 04.70.Bw, 04.20.Dw

\section{Introduction}

In a previous work a black hole solution with dilaton and monopole fields was found [1] and subsequently a generalization of this solution was given [2]. It is therefore natural to try to extend this work to the case where we have electric instead of magnetic charge and to the case where we have both charges electric and magnetic.

\footnotetext{
${ }^{1}$ E-mail: kyriakop@central.ntua.gr
} 
In this work we present two solutions one with electric charge only and another with electric and magnetic charges, and we consider special cases of these general solutions. The solutions are exact and have some very nice features. All describe static spherically symmetric black holes. Their metric is asymptotically flat, has two horizons and irreducible singularity only at $r=0$. The scalar field is singular only at $r=0$. Calculating the eigenvalues of the energy-momentum tensor $T_{\mu \nu}$ of the solutions we can show that all solutions satisfy the dominant as well as the strong energy condition outside and on their external horizon.

The solutions contain a number of parameters, which satisfy certain relations. Therefore we can pick up a number of independent parameters and express the rest in terms of them. The independent parameters will tell us if the solution is "hairy" or not. There are various formulations of the no-hair conjecture [3]-[7]. According to one of them [7] "we say that in a given theory there is black hole hair when the space-time metric and the configurations of the other fields of a stationary black hole solution are not completely specified by conserved charges defined at asymptotic infinitely".

In Einstein-Maxwell theory the stationary asymptotically flat solutions are determined by conserved charges defined at asymptotic infinity and therefore they are not "hairy" [8]. The no-hair conjecture does not generalize to theories with non-Abelian gauge fields coupled to gravity [9]. Also EinsteinMaxwell-Dilaton black hole solutions have nontrivial dilaton field with an additional charge the dilaton charge. For example the GHS-GM solution found by D. Garfinkle, G. T. Horowitz and A. Strominger [10] and previously by G. W. Gibbons and by G. W. Gibbons and K. Maeda[11] posses a dilaton charge, which however can be expressed in terms of the mass and the magnetic charge of the solution. In that sense the "hair" of the GHS-GM solution is "secondary" [12].

The solution with electric charge only we have found has three independent parameters and as such parameters we can chose the Arnowitt-DeserMisner (ADM) mass, the electric charge and an additional free parameter. Therefore according to the above definition it is a "hairy" solution. The "hair" is "primary".

The general dyonic solution and a special case of it have again three free parameters, which can be the ADM mass the magnetic charge and an additional free parameter. The electric charge is determined from the magnetic charge and it is not a free parameter. Again these solutions are "hairy" and their "hair" is "primary". Another very interesting special case of the 
general dyonic solution has as free parameters the ADM mass, the electric charge and the magnetic charge, and its "hair" is "secondary".

The thermodynamic properties of the solutions will be studied elsewhere. In the present work only their Hawking temperature is given.

\section{Lagrangian and Equations of Motion}

Consider the action

$$
\int d^{4} x \sqrt{-g} L=\int d^{4} x \sqrt{-g}\left\{R-\frac{1}{2} \partial_{\mu} \psi \partial^{\mu} \psi-f(\psi) F_{\mu \nu} F^{\mu \nu}\right\}
$$

where $R$ is the Ricci scalar, $\psi$ is a dilaton field, $f(\psi)$ is a function of $\psi$ and $F_{\mu \nu}$ is the electromagnetic field. Since we shall consider only spherically symmetric solutions having electric and magnetic charges or electric charges only we shall write

$$
F=\gamma(r) d r \wedge d t+Q_{M} \sin \theta d \theta \wedge d \phi
$$

where $\gamma(r)$ is a function of $r$ only and $Q_{M}$ is the magnetic charge of the solution. From this action we find the following equations of motion for the electromagnetic field, the dilaton field and the metric

$$
\begin{gathered}
\left(f F^{\mu \nu}\right)_{; \mu}=0 \\
\left(\partial^{\rho} \psi\right)_{; \rho}-\frac{d f}{d \psi} F_{\mu \nu} F^{\mu \nu}=0 \\
R_{\mu \nu}=\frac{1}{2} \partial_{\mu} \psi \partial_{\nu} \psi+2 f\left(F_{\mu \sigma} F_{\nu}^{\sigma}-\frac{1}{4} g_{\mu \nu} F_{\rho \sigma} F^{\rho \sigma}\right)
\end{gathered}
$$

We shall consider functions $f(\psi)$ such that if we introduce them in Eqs (3) -(5) we can solve these equations and the solutions we get are asymptotically flat, have regular horizons, have dilaton field singular only at $r=0$ and also have electric and magnetic charges or electric charge only. We write the metric in the form [10]

$$
d s^{2}=-\lambda^{2} d t^{2}+\lambda^{-2} d r^{2}+\xi^{2} d \Omega
$$

where $\lambda$ and $\xi$ are functions of $r$ only and $d \Omega=d \theta^{2}+\sin ^{2} \theta d \phi^{2}$. From Eqs (2), (3) and (6) we get

$$
\left(\xi^{2} f \gamma\right)^{\prime}=0
$$


where prime denotes differentiation with respect to $r$. From the above relation we get

$$
\gamma=\frac{Q_{E}}{\xi^{2} f}
$$

where $Q_{E}$ is an integration constant. Also since $\psi=\psi(r)$ and $F_{\mu \nu} F^{\mu \nu}=$ $-2 \gamma^{2}+\frac{2 Q_{M}^{2}}{\xi^{4}}$ the dilaton $\mathrm{Eq}(44)$, if we use Eq (8) $)$, becomes

$$
\left(\lambda^{2} \xi^{2} \psi^{\prime}\right)^{\prime}=\frac{2}{\xi^{2}} \frac{d}{d \psi}\left(\frac{Q_{E}{ }^{2}}{f}+Q_{M}^{2} f\right)
$$

The non-vanishing components of the Ricci tensor of the metric (66) are $R_{00}, R_{11}, R_{22}$ and $R_{33}=\sin ^{2} \theta R_{22}$, and for the first three components we get respectively from Eqs (2), (5) (6) and (8)

$$
\begin{gathered}
\left(\lambda^{2}\right)^{\prime \prime}+\left(\lambda^{2}\right)^{\prime}\left(\xi^{2}\right)^{\prime} \xi^{-2}=\frac{2}{\xi^{4}}\left(\frac{Q_{E}{ }^{2}}{f}+Q_{M}{ }^{2} f\right) \\
-\left(\lambda^{2}\right)^{\prime \prime} \lambda^{-2}-2\left(\xi^{2}\right)^{\prime \prime} \xi^{-2}-\left(\lambda^{2}\right)^{\prime}\left(\xi^{2}\right)^{\prime} \lambda^{-2} \xi^{-2}+\left[\left(\xi^{2}\right)^{\prime}\right]^{2} \xi^{-4}=\left(\psi^{\prime}\right)^{2} \\
-\frac{2}{\lambda^{2} \xi^{4}}\left(\frac{Q_{E}{ }^{2}}{f}+Q_{M}{ }^{2} f\right) \\
-\left[\lambda^{2}\left(\xi^{2}\right)^{\prime}\right]^{\prime}+2=\frac{2}{\xi^{2}}\left(\frac{Q_{E}{ }^{2}}{f}+Q_{M}{ }^{2} f\right)
\end{gathered}
$$

Eqs (9)-(12) form a system of four equations for the three unknowns $\lambda^{2}, \xi^{2}$ and $\psi$. Of course to get a solution we have to specify first $f=f(\psi)$. This will be done in such a way that physically interesting solutions can be found.

We have found previously [1] a solution with $\gamma=0$ and $Q_{M} \neq 0$ and later we generalized this solution [2]. In the following sections we shall find a solution with $\gamma \neq 0$ and $Q_{M}=0$ and another solution with $\gamma \neq 0$ and $Q_{M} \neq 0$, and we shall consider special cases of these solutions.

\section{Solution with Electric Field only}

Assume that

$$
f(\psi)=\left\{g_{1} e^{\left(c+\sqrt{c^{2}+1}\right) \psi}+g_{2} e^{\left(c-\sqrt{c^{2}+1}\right) \psi}\right\}^{-1}
$$

where $c, g_{1}$ and $g_{2}$ are constants, and that 


$$
Q_{M}=0
$$

Then the system of Eqs (9)-(12) has a solution with

$$
\begin{gathered}
F=Q_{E}\left\{\frac{g_{1}}{r^{2}} e^{\left(c+\sqrt{c^{2}+1}\right) \psi_{0}}+\frac{g_{2}}{(r+\alpha)^{2}} e^{\left(c-\sqrt{c^{2}+1} \psi_{0}\right.}\right\} d r \wedge d t \\
=\frac{1}{2 Q_{E}}\left\{\frac{A B\left(\sqrt{c^{2}+1}-c\right)}{\sqrt{c^{2}+1} r^{2}}+\frac{(\alpha-A)(\alpha-B)\left(\sqrt{c^{2}+1}+c\right)}{\sqrt{c^{2}+1}(r+\alpha)^{2}}\right\} d r \wedge d t \\
\lambda^{2}=\frac{(r+A)(r+B)}{r(r+\alpha)}\left(\frac{r}{r+\alpha}\right)^{\frac{c}{\sqrt{c^{2}+1}}}, \quad \xi^{2}=r(r+\alpha)\left(\frac{r+\alpha}{r}\right)^{\frac{c}{\sqrt{c^{2}+1}}} \\
e^{\psi}=e^{\psi_{0}}\left(1+\frac{\alpha}{r}\right)^{\frac{1}{\sqrt{c^{2}+1}}}
\end{gathered}
$$

where $A, B, \alpha$ and $\psi_{0}$ are integration constants, provided that the following relations are satisfied

$$
\begin{gathered}
g_{1}=\frac{A B\left(\sqrt{c^{2}+1}-c\right)}{2 Q_{E}^{2} \sqrt{c^{2}+1}} e^{-\left(c+\sqrt{c^{2}+1}\right) \psi_{0}} \\
g_{2}=\frac{(\alpha-A)(\alpha-B)\left(\sqrt{c^{2}+1}+c\right)}{2 Q_{E}^{2} \sqrt{c^{2}+1}} e^{-\left(c-\sqrt{c^{2}+1}\right)} \psi_{0}
\end{gathered}
$$

From Eqs ([6) and (16) we get

$$
\begin{gathered}
d s^{2}=-\frac{(r+A)(r+B)}{r(r+\alpha)}\left(\frac{r}{r+\alpha}\right)^{\frac{c}{\sqrt{c^{2}+1}}} d t^{2}+\frac{r(r+\alpha)}{(r+A)(r+B)}\left(\frac{r+\alpha}{r}\right)^{\frac{c}{\sqrt{c^{2}+1}}} d r^{2} \\
+r(r+\alpha)\left(\frac{r+\alpha}{r}\right)^{\frac{c}{\sqrt{c^{2}+1}}} d \Omega
\end{gathered}
$$

Our solution is given by Eqs (15) and (17)-(20) .

Eq (15) indicates that we have a point charge located at the origin and a charge distribution, which is spherically symmetric with respect to the origin.

Eqs (17) and (20) have appeared in a previous work [2] and therefore some of the arguments presented there hold also in the present case. From Eq (20) we get asymptotically

$$
-g_{00}=1-\frac{\alpha\left(1+\frac{c}{\sqrt{c^{2}+1}}\right)-A-B}{r}+O\left(r^{-2}\right)
$$


which means that the solution is asymptotically flat and its ADM mass $M$ is given by

$$
2 M=\alpha\left(1+\frac{c}{\sqrt{c^{2}+1}}\right)-A-B
$$

Eq (17) tells us that $\psi_{0}$ is the asymptotic value of $\psi$. Also we shall make the choice

$$
\alpha>0, \quad A<0, \quad B<0,
$$

for which $\psi$ is singular only at $r=0$ and for which we have

$$
g_{1}>0, \quad g_{2}>0, \quad M>0
$$

The solution has the integration constants $\alpha, A, B, \psi_{0}$ and $Q_{E}$, which for given $g_{1}, g_{2}$ and $c$ must satisfy Eqs (18) and (19). Therefore only three of them are independent. Introducing the ADM mass $M$ by the relation (22) we can take $M, Q_{E}$ and $\psi_{0}$ as independent parameters. Therefore our solution has arbitrary mass, arbitrary electric charge and an additional arbitrary parameter. Thus it is a "hairy" solution according to the definition given in Ref. [7. The "hair" is "primary".

If (23) hold our metric is singular at $r=-A$ and $r=-B$ but not at $r=-\alpha$. However the Ricci scalar $R$ and the curvature scalar $R_{\mu \nu \rho \sigma} R^{\mu \nu \rho \sigma}$ are not singular at $r=-A$ and $r=-B$. Indeed we have [2]

$$
\begin{gathered}
R=\frac{\alpha^{2}(r+A)(r+B)}{2\left(c^{2}+1\right) r^{3}(r+\alpha)^{3}}\left(\frac{r}{r+\alpha}\right)^{\frac{c}{\sqrt{c^{2}+1}}} \\
R_{\mu \nu \rho \sigma} R^{\mu \nu \rho \sigma}=\frac{P(r, \alpha, A, B)}{4\left(c^{2}+1\right)^{2} r^{6}(r+\alpha)^{6}}\left(\frac{r}{r+\alpha}\right)^{\frac{2 c}{\sqrt{c^{2}+1}}}
\end{gathered}
$$

where $P(r, \alpha, A, B)$ is a complicated polynomial of $r, \alpha A$ and $B$. Therefore we have an irremovable singularity only at $r=0$.

Proceeding as in Ref. [1] and 2] we can introduce Eddington- Finkelstein type coordinates and we can show that we have a black hole solution with two horizons located at $r=-A$ and $r=-B$.

The energy-momentum tensor $T_{\mu \nu}$ of our solution is given by

$$
\begin{gathered}
T_{\mu \nu}=\partial_{\mu} \psi \partial_{\nu} \psi+4 f F_{\mu \rho} F_{\nu}{ }^{\rho}-g_{\mu \nu}\left\{\frac{1}{2} \partial_{\rho} \psi \partial^{\rho} \psi+f F_{\rho \sigma} F^{\rho \sigma}\right\} \\
=\frac{\alpha^{2}}{\left(c^{2}+1\right) r^{2}(r+\alpha)^{2}}\left(\delta_{\mu r} \delta_{\nu r}-\frac{1}{2} g_{\mu \nu} g^{r r}\right)+\frac{2}{r^{2}(r+\alpha)^{2}}\left\{\frac{A B\left(\sqrt{c^{2}+1}-c\right)}{\sqrt{c^{2}+1}}\right.
\end{gathered}
$$




$$
\begin{gathered}
\left.\times\left(\frac{r+\alpha}{r}\right)^{\frac{c+\sqrt{c^{2}+1}}{\sqrt{c^{2}+1}}}+\frac{(\alpha-A)(\alpha-B)\left(\sqrt{c^{2}+1}+c\right)}{\sqrt{c^{2}+1}}\left(\frac{r+\alpha}{r}\right)^{\frac{c-\sqrt{c^{2}+1}}{\sqrt{c^{2}+1}}}\right\} \\
\times\left\{-\frac{r(r+\alpha)}{(r+A)(r+B)}\left(\frac{r+\alpha}{r}\right)^{\frac{c}{\sqrt{c^{2}+1}}} \delta_{\mu r} \delta_{\nu r}\right. \\
\left.+\frac{(r+A)(r+B)}{r(r+\alpha)}\left(\frac{r}{r+\alpha}\right)^{\frac{c}{\sqrt{c^{2}+1}}} \delta_{\mu t} \delta_{\nu t}+\frac{g_{\mu \nu}}{2}\right\}
\end{gathered}
$$

Calculating the eigenvalues of $T_{\mu \nu}$ we can show that it satisfies the dominant as well as the strong energy condition outside and on the external horizon.

The Hawking temperature $T_{H}$ of our solution if $A<B<0$ and $\alpha>0$ is given by 13

$$
T_{H}=\frac{\left|\lambda^{2^{\prime}}(-A)\right|}{4 \pi}=\frac{(B-A)(-A)^{-1+\frac{c}{\sqrt{c^{2}+1}}}(\alpha-A)^{-1-\frac{c}{\sqrt{c^{2}+1}}}}{4 \pi}
$$

We argued before that we can take $M, Q_{E}$ and $\psi_{0}$ as independent parameters of the solution. In this case using Eqs (18), (19) and (22) we can express $A, B$ and $\alpha$ in terms of $M, Q_{E}$ and $\psi_{0}$. For $A \leq B<0, \alpha>0$ and assuming for simplicity that $c=0$ we get

$$
\begin{gathered}
A=\frac{1}{2 M}\left\{Q_{E}^{2}\left(g_{2} e^{-\psi_{0}}-g_{1} e^{\psi_{0}}\right)-2 M^{2}\right. \\
\left.-\sqrt{\left[Q_{E}^{2}\left(g_{2} e^{-\psi_{0}}-g_{1} e^{\psi_{0}}\right)-2 M^{2}\right]^{2}-8 M^{2} Q_{E}^{2} g_{1} e_{0}^{\psi}}\right\} \\
B=\frac{1}{2 M}\left\{Q_{E}^{2}\left(g_{2} e^{-\psi_{0}}-g_{1} e^{\psi_{0}}\right)-2 M^{2}\right. \\
\left.+\sqrt{\left[Q_{E}^{2}\left(g_{2} e^{-\psi_{0}}-g_{1} e^{\psi_{0}}\right)-2 M^{2}\right]^{2}-8 M^{2} Q_{E}^{2} g_{1} e_{0}^{\psi}}\right\} \\
\alpha=\frac{Q_{E}^{2}}{M}\left(g_{2} e^{-\psi_{0}}-g_{1} e^{\psi_{0}}\right)
\end{gathered}
$$

where

$$
\begin{gathered}
2 M^{2} \geq Q_{E}^{2}\left(\sqrt{g_{2}} e^{-\frac{\psi_{0}}{2}}+\sqrt{g_{1}} e^{\frac{\psi_{0}}{2}}\right)^{2} \\
g_{2} e^{-\psi_{0}}-g_{1} e^{\psi_{0}}>0
\end{gathered}
$$

We have for the extremal solution $A=B<0$ and $\alpha>0$. This happens if Eq (32) with the equality sign and Eq. (33) hold. In this case we get

$$
A=B=-\sqrt{2 g_{1}} Q_{E} e^{\frac{\psi_{0}}{2}}
$$


Also if $A>0, B>0$ and $\alpha>0$ the metric has a naked singularity at $r=0$. This happens if

$$
2 M^{2}<Q_{E}^{2}\left(\sqrt{g_{2}} e^{-\frac{\psi_{0}}{2}}-\sqrt{g_{1}} e^{\frac{\psi_{0}}{2}}\right)^{2} \text { and } g_{2} e^{-\psi_{0}}-g_{1} e^{\psi_{0}}>0
$$

Finally if $g_{1}=1$ and $g_{2}=0$ our solution reduces to the GHS-GM solution for electric charge found by D. Garfinkle, G. T. Horowitz and A. Strominger [10, and by M. Rakhmanov[14, and previously by G. W. Gibbons and by G. W. Gibbons and K. Maeda[11].

\section{Dyonic Solution}

Let us take

$$
\begin{gathered}
f_{ \pm}(\psi)=g_{1}^{\prime} e^{\left(c+\sqrt{c^{2}+1}\right) \psi}+g_{2}^{\prime} e^{\left(c-\sqrt{c^{2}+1}\right) \psi} \\
\pm \sqrt{\left[g_{1}^{\prime} e^{\left(c+\sqrt{c^{2}+1}\right) \psi}+g_{2}^{\prime} e^{\left(c-\sqrt{c^{2}+1}\right) \psi}\right]^{2}+g_{3}}
\end{gathered}
$$

where $c, g_{1}^{\prime}, g_{2}^{\prime}$ and $g_{3}$ are real constants. Then the system of Eqs (91)-(12) has a solution with

$$
\begin{aligned}
& F_{ \pm}=\frac{Q_{E}}{\xi^{2} f_{ \pm}} d r \wedge d t+Q_{M} \sin \theta d \theta \wedge d \phi=\frac{Q_{M}^{2} f_{\mp}}{Q_{E} \xi^{2}} d r \wedge d t+Q_{M} \sin \theta d \theta \wedge d \phi \\
&= \frac{Q_{M}^{2} e^{c \psi_{0}}}{Q_{E}}\left\{\frac{g_{1}^{\prime} e^{\sqrt{c^{2}+1} \psi_{0}}}{r^{2}}+\frac{g_{2}^{\prime} e^{-\sqrt{c^{2}+1}} \psi_{0}}{(r+\alpha)^{2}} \mp\left[\frac{g_{1}^{\prime 2} e^{2 \sqrt{c^{2}+1}} \psi_{0}}{r^{4}}+\frac{g_{2}^{\prime 2} e^{-2 \sqrt{c^{2}+1}} \psi_{0}}{(r+\alpha)^{4}}\right.\right. \\
&+\left.\left.\frac{2 g_{1}^{\prime} g_{2}^{\prime}}{r^{2}(r+\alpha)^{2}}+\frac{g_{3} e^{-2 c \psi_{0}}}{r^{2}(r+\alpha)^{2}}\left(\frac{r}{r+\alpha}\right)^{\frac{2 c}{\sqrt{c^{2}+1}}}\right]^{\frac{1}{2}}\right\} d r \wedge d t+Q_{M} \sin \theta d \theta \wedge d t \\
&= \frac{1}{4 \sqrt{c^{2}+1} Q_{E}}\left\{\frac{A B\left(\sqrt{c^{2}+1}-c\right)}{r^{2}}+\frac{(\alpha-A)(\alpha-B)\left(\sqrt{c^{2}+1}+c\right)}{(r+\alpha)^{2}}\right. \\
& \mp\left[\frac{A^{2} B^{2}\left(\sqrt{c^{2}+1}-c\right)^{2}}{r^{4}}+\frac{(\alpha-A)^{2}(\alpha-B)^{2}\left(\sqrt{c^{2}+1}+c\right)^{2}}{(r+\alpha)^{4}}+\right. \\
&\left.\left.\frac{2 A B(\alpha-A)(\alpha-B)}{r^{2}(r+\alpha)^{2}}-\frac{16 Q_{E}^{2} Q_{M}^{2}\left(c^{2}+1\right)}{r^{2}(r+\alpha)^{2}}\left(\frac{r}{r+\alpha}\right)^{\frac{2 c}{\sqrt{c^{2}+1}}}\right]^{\frac{1}{2}}\right\} d r \wedge d t+Q_{M} \sin \theta d \theta \wedge d t
\end{aligned}
$$


and with $\lambda^{2}, \xi^{2}$ and $\psi$ given by Eqs (16) and 17), where $A, B, \alpha, \psi_{0}, Q_{E}$ and $Q_{M}$ are constants, which are connected with the given constants $g_{1}^{\prime}, g_{2}^{\prime}$, $g_{3}$ and $c$ with the relations

$$
\begin{gathered}
g_{1}^{\prime}=\frac{A B\left(\sqrt{c^{2}+1}-c\right)}{4 Q_{M}^{2} \sqrt{c^{2}+1}} e^{-\left(c+\sqrt{c^{2}+1}\right)} \psi_{0} \\
g_{2}^{\prime}=\frac{(\alpha-A)(\alpha-B)\left(\sqrt{c^{2}+1}+c\right)}{4 Q_{M}^{2} \sqrt{c^{2}+1}} e^{-\left(c-\sqrt{c^{2}+1}\right)} \psi_{0} \\
g_{3}=-\frac{Q_{E}^{2}}{Q_{M}^{2}}
\end{gathered}
$$

The charges $Q_{E}$ and $Q_{M}$ are connected by the previous relation, which means that it is not possible to take arbitrary values both. Our most general dyonic solution is given by Eqs (17), (20) and (37)-(40)

The ADM mass $M$ of the solution is given by Eq (22) and for the choice (23), which we shall make again, $\psi$ is singular only at $r=0$ and we get

$$
g_{1}^{\prime}>0, \quad g_{2}^{\prime}>0, \quad M>0
$$

The solution has a number of constants namely the magnetic charge $Q_{M}$ and the integration constants $A, B, \alpha, \psi_{0}$ and $Q_{E}$, which for given $g_{1}^{\prime}, g_{2}^{\prime}$, $g_{3}$ and $c$ must satisfy Eqs (38)-(40). Therefore only three of them are independent and if we take into account Eq. (22) we can choose $M, Q_{M}$ and $\psi_{0}$ as independent parameters. Thus the solution has arbitrary mass, arbitrary magnetic charge and an additional arbitrary parameter. Therefore it is a "hairy" solution according to the definition given in Ref. [7]. The "hair" is "primary".

Since the Ricci scalar $R$ and the curvature scalar $R_{\mu \nu \rho \sigma} R^{\mu \nu \rho \sigma}$ of the solution are given by Eqs (25) and (26) the singularities at $r=-A$ and $r=-B$ are coordinate singularities and only the singularity at $r=0$ is irremovable. We have a black hole solution with two horizons at $r=-A$ and $r=-B$.

The energy-momentum tensor $T_{\mu \nu}$ of our solution is given by

$$
\begin{gathered}
T_{\mu \nu}=\partial_{\mu} \psi \partial_{\nu} \psi+4 f_{ \pm} F_{\mu \rho} F_{\nu}{ }^{\rho}-g_{\mu \nu}\left\{\frac{1}{2} \partial_{\rho} \psi \partial^{\rho} \psi+f_{ \pm} F_{\rho \sigma} F^{\rho \sigma}\right\} \\
=\frac{\alpha^{2}}{\left(c^{2}+1\right) r^{2}(r+\alpha)^{2}} \delta_{\mu r} \delta_{\nu r}+\frac{h_{\mp}}{\sqrt{c^{2}+1} r(r+\alpha)}
\end{gathered}
$$




$$
\begin{gathered}
\times\left\{\frac{(r+A)(r+B)}{r(r+\alpha)}\left(\frac{r}{r+\alpha}\right)^{\frac{c}{\sqrt{c^{2}+1}}} \delta_{\mu t} \delta_{\nu t}-\frac{r(r+\alpha)}{(r+A)(r+B)}\left(\frac{r+\alpha}{r}\right) \frac{c}{\sqrt{c^{2}+1}} \delta_{\mu r} \delta_{\nu r}\right\} \\
+\frac{h_{ \pm}}{\sqrt{c^{2}+1}}\left(\frac{r}{r+\alpha}\right)^{\frac{c}{\sqrt{c^{2}+1}}}\left(\delta_{\mu \theta} \delta_{\nu \theta}+\sin ^{2} \theta \delta_{\mu \phi} \delta_{\nu \phi}\right)-\frac{g_{\mu \nu}}{2}\left\{\frac{\alpha^{2}(r+A)(r+B)}{\left(c^{2}+1\right) r^{3}(r+\alpha)^{3}}\right. \\
\left.-\frac{h_{\mp}}{\sqrt{c^{2}+1} r(r+\alpha)}+\frac{h_{ \pm}}{\sqrt{c^{2}+1} r(r+\alpha)}\left(\frac{r}{r+\alpha}\right)^{\frac{2 c}{\sqrt{c^{2}+1}}}\right\}
\end{gathered}
$$

where

$$
\begin{aligned}
h_{ \pm} & =4 \sqrt{c^{2}+1} Q_{M}^{2}\left(\frac{r+\alpha}{r}\right) \frac{c}{\sqrt{c^{2}+1}}\left\{g_{1}^{\prime} \frac{e^{\left(c+\sqrt{c^{2}+1}\right)} \psi_{0}}{r^{2}}+g_{2}^{\prime} \frac{e^{\left(c-\sqrt{c^{2}+1}\right) \psi_{0}}}{(r+\alpha)^{2}}\right. \\
& \left. \pm\left[\left\{g_{1}^{\prime} \frac{e^{\left(c+\sqrt{c^{2}+1}\right)} \psi_{0}}{r^{2}}+g_{2}^{\prime} \frac{e^{\left(c-\sqrt{c^{2}+1}\right)} \psi_{0}}{(r+\alpha)^{2}}\right\}^{2}+g_{3}\left(\frac{r}{r+\alpha}\right)^{\frac{2 c}{\sqrt{c^{2}+1}}}\right]^{\frac{1}{2}}\right\}
\end{aligned}
$$

or

$$
\begin{gathered}
h_{ \pm}=\frac{A B\left(\sqrt{c^{2}+1}-c\right)}{r^{2}}\left(\frac{r+\alpha}{r}\right)^{\frac{c}{\sqrt{c^{2}+1}}}+\frac{(\alpha-A)(\alpha-B)\left(\sqrt{c^{2}+1}+c\right)}{(r+\alpha)^{2}} \\
\times\left(\frac{r}{r+\alpha}\right)^{\frac{c}{\sqrt{c^{2}+1}}} \pm\left\{\left[\frac{A B\left(\sqrt{c^{2}+1}-c\right)}{r^{2}}\left(\frac{r+\alpha}{r}\right)^{\frac{c}{\sqrt{c^{2}+1}}}\right.\right. \\
\left.\left.+\frac{(\alpha-A)(\alpha-B)\left(\sqrt{c^{2}+1}+c\right)}{(r+\alpha)^{2}}\left(\frac{r}{r+\alpha}\right)^{\frac{c}{\sqrt{c^{2}+1}}}\right]^{2}-\frac{16\left(c^{2}+1\right) Q_{E}^{2} Q_{M}^{2}}{r^{2}(r+\alpha)^{2}}\right\}^{\frac{1}{2}}
\end{gathered}
$$

Calculating the eigenvalues of $T_{\mu \nu}$ we can show that it satisfies the dominant as well as the strong energy condition outside and on the external horizon.

The Hawking temperature $T_{H}$ of our solution if $A<B<0$ and $\alpha>0$ is given by Eq. (28).

If we chose $M, Q_{M}$ and $\psi_{0}$ as free parameters using Eqs (22) (38) and (39) we can express $A, B$ and $\alpha$ in terms of $M, Q_{M}$ and $\psi_{0}$. To simplify the calculations we shall assume that $c=0$ and we shall call $g_{1}^{\prime \prime}$ and $g_{2}^{\prime \prime}$ the expressions $g_{1}^{\prime}$ and $g_{2}^{\prime}$ of Eqs (38) and (39) for $c=0$ i. e.

$$
g_{1}^{\prime}(c=0) \equiv g_{1}^{\prime \prime}=\frac{A B}{4 Q_{M}^{2}} e^{-\psi_{0}} \quad g_{2}^{\prime}(c=0) \equiv g_{2}^{\prime \prime}=\frac{(\alpha-A)(\alpha-B)}{4 Q_{M}^{2}} e^{\psi_{0}}
$$

Then for $A \leq B<0$ and $\alpha>0$ we find that

$$
A=\frac{1}{M}\left\{Q_{M}^{2}\left(g_{2}^{\prime \prime} e^{-\psi_{0}}-g_{1}^{\prime \prime} e^{\psi_{0}}\right)-M^{2}\right.
$$




$$
\begin{gathered}
\left.-\sqrt{\left[Q_{M}^{2}\left(g_{2}^{\prime \prime} e^{-\psi_{0}}-g_{1}^{\prime \prime} e^{\psi_{0}}\right)-M^{2}\right]^{2}-4 M^{2} Q_{M}^{2} g_{1}^{\prime \prime} e^{\psi_{0}}}\right\} \\
B=\frac{1}{M}\left\{Q_{M}^{2}\left(g_{2}^{\prime \prime} e^{-\psi_{0}}-g_{1}^{\prime \prime} e^{\psi_{0}}\right)-M^{2}\right. \\
\left.+\sqrt{\left[Q_{M}^{2}\left(g_{2}^{\prime \prime} e^{-\psi_{0}}-g_{1}^{\prime \prime} e^{\psi_{0}}\right)-M^{2}\right]^{2}-4 M^{2} Q_{M}^{2} g_{1}^{\prime \prime} e^{\psi_{0}}}\right\} \\
\alpha=\frac{2 Q_{M}^{2}}{M}\left(g_{2}^{\prime \prime} e^{-\psi_{0}}-g_{1}^{\prime \prime} e^{\psi_{0}}\right)
\end{gathered}
$$

and that we must have

$$
\begin{gathered}
M^{2} \geq Q_{M}^{2}\left(\sqrt{g_{2}^{\prime \prime}} e^{-\frac{\psi_{0}}{2}}+\sqrt{g_{1}^{\prime \prime}} e^{\frac{\psi_{0}}{2}}\right)^{2} \\
g_{2}^{\prime \prime} e^{-\psi_{0}}-g_{1}^{\prime \prime} e^{\psi_{0}}>0
\end{gathered}
$$

We get the extremal solution if $A=B<0$ and $\alpha>0$. This happens if relation (49) with the equality sign and relation (50) hold. Also if $A>0$, $B>0$ and $\alpha>0$ the metric has a naked singularity at $r=0$. This happens if

$$
M^{2}<Q_{M}^{2}\left(\sqrt{g_{2}^{\prime \prime}} e^{-\frac{\psi_{0}}{2}}-\sqrt{g_{1}^{\prime \prime}} e^{\frac{\psi_{0}}{2}}\right)^{2}
$$

A very interesting dyonic solution is obtained if in the above general dyonic solution given by Eqs (36), (17), (20) and (37)-(40) we put

$$
c=0, \quad g_{2}^{\prime \prime}=\frac{1}{2} \quad \text { and } \quad g_{3}=-4 g_{1}^{\prime \prime} g_{2}^{\prime \prime}
$$

where $g_{1}^{\prime \prime}$ and $g_{2}^{\prime \prime}$ denote the values of $g_{1}^{\prime}$ and $g_{2}^{\prime}$ if $c=0$. Then Eq (36) gives

$$
f_{-}(\psi)=e^{-\psi}
$$

and Eqs (17), (20) and (37)-(40) become

$$
\begin{gathered}
e^{\psi}=e^{\psi_{0}}\left(1+\frac{\alpha}{r}\right) \\
d s^{2}=-\frac{(r+A)(r+B)}{r(r+\alpha)} d t^{2}+\frac{r(r+\alpha)}{(r+A)(r+B)} d r^{2}+r(r+\alpha) d \Omega \\
F_{-}=\frac{Q_{E} e^{\psi_{0}}}{r^{2}} d r \wedge d t+Q_{M} \sin \theta d \theta \wedge d \phi
\end{gathered}
$$




$$
\frac{A B e^{-\psi_{0}}}{2 Q_{E}{ }^{2}}=\frac{(\alpha-A)(\alpha-B) e^{\psi_{0}}}{2 Q_{M}{ }^{2}}=1
$$

That is if in the Lagrangian of Eq. (11) we have $f(\psi)=f_{-}(\psi)=e^{-\psi}$ Eqs (54)-(56), where the constants $A, B, \alpha, \psi_{0}, Q_{E}$ and $Q_{M}$ satisfy the relations (57), give a dyonic solution. Eq. (56) tells us that the electromagnetic field is created by a point electric charge $Q_{E} e^{\psi_{0}}$ and a point magnetic charge $Q_{M}$, which are located at the origin.

The ADM mass of the solution is given by Eq. (22) with $c=0$ i.e.

$$
2 M=\alpha-A-B
$$

Also $\psi_{0}$ is the asymptotic value of $\psi$ and for the choice

$$
\alpha>0, \quad A<0, \quad B<0
$$

which is consistent with the constraint Eqs (57) and for which $2 M>0$, the metric is singular at $r=0, r=-A$ and $r=-B$. However the Ricci scalar $R$ and the curvature scalar $R_{\mu \nu \rho \sigma} R^{\mu \nu \rho \sigma}$, which are given by Eqs (25) and (26) with $c=0$, are singular only at $r=0$. This means that the singularities at $r=-A$ and $r=-B$ are coordinate singularities and only at $r=0$ we have an irremovable singularity. The solution is a black hole solution with two horizons located at $r=-A$ and $r=-B$. Also since $\alpha>0$ the dilaton field $\psi$ is singular only at $r=0$.

The action (11) if $f(\psi)$ is the $f_{-}(\psi)$ of Eq. (53) is invariant under the transformation

$$
g_{\mu \nu} \rightarrow g_{\mu \nu}, \quad \psi \rightarrow \psi+b, \quad F_{\mu \nu} \rightarrow e^{\frac{b}{2}} F \mu \nu
$$

where $\mathrm{b}$ is a constant.This invariance leads to the conserved dilaton current

$$
J_{\mu}=\partial_{\mu} \psi+2 e^{-\psi} F_{\mu \nu} A^{\nu}
$$

where $A^{\nu}$ is the vector potential, from which we get the conserved dilaton charge

$$
D=\frac{1}{4 \pi} \int_{S} J_{\mu} d S^{\mu}=-\alpha
$$

where the integral is over a two-sphere at spatial infinity. In deriving the above expression for the dilaton charge we have assumed that $A^{t}$ vanishes at 
infinity. Since the transformation (60) changes $\psi_{0}$ we can give it any value we want, for example the value zero. Therefore $\psi_{0}$ cannot be considered as a parameter of the model. This means that we have only three free parameters, since we have the parameters $A, B, \alpha, Q_{M}$ and $Q_{E}$ which must satisfy Eqs (57). We shall choose as free parameters the mass $M$ and the electric and magnetic charges $Q_{E}$ and $Q_{M}$.

If we define $Q_{E}^{\prime}$ and $Q_{M}^{\prime}$ by the relations

$$
Q_{E}^{\prime}=Q_{E} e^{\frac{\psi_{0}}{2}}, \quad Q_{M}^{\prime}=Q_{M} e^{\frac{-\psi_{0}}{2}}
$$

and solve Eqs (57) and (58) for $A, B$ and $\alpha$ in terms of $M, Q_{E}^{\prime}$ and $Q_{M}^{\prime}$ assuming that $A \leq B<0$ and $\alpha>0$ we find

$$
\begin{gathered}
A=\frac{1}{2 M}\left\{{Q_{M}^{\prime}}^{2}-{Q_{E}^{\prime}}^{2}-2 M^{2}-\sqrt{\left({Q_{M}^{\prime}}^{2}-{Q_{E}^{\prime}}^{2}-2 M^{2}\right)^{2}-8 M^{2}{Q_{E}^{\prime}}^{2}}\right\} \\
B=\frac{1}{2 M}\left\{{Q_{M}^{\prime}}^{2}-{Q_{E}^{\prime}}^{2}-2 M^{2}+\sqrt{\left({Q_{M}^{\prime}}^{2}-{Q_{E}^{\prime}}^{2}-2 M^{2}\right)^{2}-8 M^{2}{Q_{E}^{\prime}}^{2}}\right\} \\
\alpha=\frac{1}{M}\left({Q_{M}^{\prime}}^{2}-{Q_{E}^{\prime}}^{2}\right)
\end{gathered}
$$

and that we must have

$$
\sqrt{2} M \geq\left|Q_{M}^{\prime}\right|+\left|Q_{E}^{\prime}\right|
$$

and

$$
\left|Q_{M}^{\prime}\right|-\left|Q_{E}^{\prime}\right|>0
$$

Using Eqs (62) and (66) we can express the dilaton charge $D$ in terms of $Q_{E}^{\prime}$ and $Q_{M}^{\prime}$. We get

$$
D=\frac{1}{M}\left(Q_{E}^{\prime 2}-Q_{M}^{\prime 2}\right)
$$

We have for the extremal solution $A=B<0$ and $\alpha>0$. This happens if Eq. (67) with the equality sign and Eq. (68) hold. Also if $A>0, B>0$ and $\alpha>0$ the metric has a naked singularity at $r=0$. This happens if

$$
\sqrt{2} M<\left|Q_{M}^{\prime}\right|-\left|Q_{E}^{\prime}\right|
$$

If $Q_{M}^{\prime}=0$ the previous solution becomes the the GHS-GM solution for electric charge only. Also if $Q_{E}^{\prime}=0$ this solution becomes

$$
d s^{2}=\left(1-\frac{2 M}{r+\frac{Q_{M}^{\prime 2}}{r}}\right) d t^{2}+\left(1-\frac{2 M}{r+\frac{Q_{M}^{\prime}{ }^{2}}{r}}\right)^{-1} d r^{2}+r\left(r+\frac{Q_{M}^{\prime}{ }^{2}}{r}\right) d \Omega
$$




$$
e^{\psi}=e^{\psi_{0}} \frac{r+\frac{Q_{M}^{\prime}{ }^{2}}{r}}{r}
$$

and if we replace $r$ by $r^{\prime}=r+\frac{{Q_{M}^{\prime}}^{2}}{r}$ we get the GHS-GM solution for magnetic charge.

The energy-momentum tensor $T_{\mu \nu}$ of the solution given by Eqs (54)-(57) is

$$
\begin{gathered}
T_{\mu \nu}=\partial_{\mu} \psi \partial_{\nu} \psi+4 e^{-\psi} F_{\mu \rho} F_{\nu}{ }^{\rho}-g_{\mu \nu}\left\{\frac{1}{2} \partial_{\rho} \psi \partial^{\rho} \psi+e^{-\psi} F_{\rho \sigma} F^{\rho \sigma}\right\} \\
=\frac{\alpha^{2}}{r^{2}(r+\alpha)^{2}} \delta_{\mu r} \delta_{\nu r}+\frac{4 Q_{E}^{\prime 2}}{r(r+\alpha)^{3}}\left\{\frac{(r+A)(r+B)}{r(r+\alpha)} \delta_{\mu t} \delta_{\nu t}-\frac{r(r+\alpha)}{(r+A)(r+B)} \delta_{\mu r} \delta_{\nu r}\right\} \\
+\frac{4 Q_{M}^{\prime 2}}{r^{2}}\left(\delta_{\mu \theta} \delta_{\nu \theta}+\sin ^{2} \theta \delta_{\mu \phi} \delta_{\nu \phi}\right)-g_{\mu \nu}\left\{\frac{\alpha^{2}(r+A)(r+B)}{2 r^{3}(r+\alpha)^{3}}-\frac{2 Q_{E}^{\prime 2}}{r(r+\alpha)^{3}}+\frac{2 Q_{M}^{\prime 2}}{r^{3}(r+\alpha)}\right\}
\end{gathered}
$$

Calculating the eigenvalues of $T_{\mu \nu}$ we can show that it satisfies the dominant as well as the strong energy condition outside and on the external horizon.

The solution given by Eqs (54)-(57) has for $A<B<0$ and $\alpha>0$ the Hawking temperature

$$
\begin{gathered}
T_{H}=\frac{\left|\lambda^{2^{\prime}}(-A)\right|}{4 \pi}=\frac{B-A}{4 \pi A(A-\alpha)} \\
=\frac{\sqrt{\left(2 M^{2}-{Q_{E}^{\prime}}^{2}-Q_{M}^{\prime}{ }^{2}\right)^{2}-4 Q_{E}^{\prime 2} Q_{M}^{\prime}{ }^{2}}}{4 \pi M\left\{\sqrt{\left(2 M^{2}-{Q_{E}^{\prime}}^{2}-{Q_{M}^{\prime}}^{2}\right)^{2}-4{Q_{E}^{\prime}}^{2}{Q_{M}^{\prime}}^{2}}+2 M^{2}-{Q_{E}^{\prime}}^{2}-{Q_{M}^{\prime}}^{2}\right\}}
\end{gathered}
$$

Also in the general dyonic solution we can put

$$
c=0, \quad g_{1}^{\prime \prime}=\frac{1}{2} \quad \text { and } \quad g_{3}=-4 g_{1}^{\prime \prime} g_{2}^{\prime \prime}
$$

where as before $g_{1}^{\prime \prime}$ and $g_{2}^{\prime \prime}$ denote the values of $g_{1}^{\prime}$ and $g_{2}^{\prime}$ if $c=0$. Then Eq (36) gives

$$
f_{+}(\psi)=e^{\psi}
$$

and Eqs (17), (20) and (37)-(140) become

$$
\begin{gathered}
e^{\psi}=e^{\psi_{0}}\left(1+\frac{\alpha}{r}\right) \\
d s^{2}=-\frac{(r+A)(r+B)}{r(r+\alpha)} d t^{2}+\frac{r(r+\alpha)}{(r+A)(r+B)} d r^{2}+r(r+\alpha) d \Omega
\end{gathered}
$$




$$
\begin{gathered}
F_{+}=\frac{Q_{E} e^{-\psi_{0}}}{(r+\alpha)^{2}} d r \wedge d t+Q_{M} \sin \theta d \theta \wedge d \phi \\
\frac{A B e^{-\psi_{0}}}{2 Q_{M}{ }^{2}}=\frac{(\alpha-A)(\alpha-B) e^{\psi_{0}}}{2 Q_{E}{ }^{2}}=1
\end{gathered}
$$

In the above solution let us make the replacements

$$
\begin{gathered}
r \rightarrow r^{\prime}=r+\alpha, \quad A \rightarrow A^{\prime}=A-\alpha, \quad B \rightarrow B^{\prime}=B-\alpha, \\
\alpha \rightarrow \alpha^{\prime}=-\alpha, \quad \psi \rightarrow \psi^{\prime}=-\psi, \quad \psi_{0} \rightarrow \psi_{0}^{\prime}=-\psi_{0}
\end{gathered}
$$

Then we find that the solution given by Eqs (77)-(80) in the primed quantities is identical to the solution given by Eqs (54)- (57)

Finally in the general dyonic solution let as put

$$
c=0 \text { and } 2 g_{1}^{\prime \prime} g_{2}^{\prime \prime}+g_{3}=0
$$

where $g_{1}^{\prime \prime}$ and $g_{2}^{\prime \prime}$ denote the values of $g_{1}^{\prime}$ and $g_{2}^{\prime}$ if $c=0$. Then Eq. (36) gives

$$
f_{ \pm}(\psi)=g_{1}^{\prime \prime} e^{\psi}+g_{2}^{\prime \prime} e^{-\psi} \pm \sqrt{g_{1}^{\prime \prime 2} e^{2 \psi}+g_{2}^{\prime \prime 2} e^{-2 \psi}}
$$

Eqs (17), (20), (38) and (39) become Eqs (154), (155) and (45), and Eqs (37) and (40) take respectively the forms

$$
\begin{gathered}
F_{ \pm}=\frac{Q_{E}}{\xi^{2} f_{ \pm}} d r \wedge d t+Q_{M} \sin \theta d \theta \wedge d \phi=\frac{Q_{M}^{2}}{Q_{E}}\left\{g_{1}^{\prime \prime} \frac{e^{\psi_{0}}}{r^{2}}\right. \\
\left.+g_{2}^{\prime \prime} \frac{e^{-\psi_{0}}}{(r+\alpha)^{2}} \mp \sqrt{g_{1}^{\prime 2} \frac{e^{2 \psi_{0}}}{r^{4}}+g_{2}^{\prime \prime 2} \frac{e^{-2 \psi_{0}}}{(r+\alpha)^{4}}}\right\} d r \wedge d t+Q_{M} \sin \theta \wedge d \phi \\
Q_{E}{ }^{2}=2 g_{1}^{\prime \prime} g_{2}^{\prime \prime} Q_{M}{ }^{2}
\end{gathered}
$$

where $g_{1}^{\prime \prime}$ and $g_{2}^{\prime \prime}$ are given by Eqs (45). If we use Eq. (85) to determine $Q_{E}$ we can say that we have the parameters $A, B, \alpha, \psi_{0}$ and $Q_{M}$, which must satisfy Eqs (45). Therefore if we introduce the ADM mass $M$ by Eq. (58) we can take $M, Q_{M}$ and $\psi_{0}$ as free parameters, and express $A, B$ and $\alpha$ by Eqs (46) - (48). Then according to Ref. [7] the above is a "hairy" solution. The "hair" is "primary". 


\section{References}

[1] E. Kyriakopoulos, submitted for publication in Int. J. Mod. Phys. D, December 2006 Special Issue.

[2] E. Kyriakopoulos, Black Hole in a Model with Dilaton and Monopole Fields II, NTUA preprint

[3] R. Ruffini and J. A. Wheeler, Phys. Today 24 (1),30 (1971).

[4] J. D. Bekenstein, Phys. Rev. D 5, 1239 (1972), 5, 2403 (1972), 51 R6608 (1995), gr-gc/9808028,

[5] S. Coleman, J. Preskill and F. Wilczek, Nucl. Phys. B 378, 175 (1992).

[6] P. Bizon, Acta Phys. Pol. B 22, 877 (1994), gr-gc/9402016,

[7] D. Nunez, H. Quevedo and D. Sudarsky, Phys. Rev. Lett. 76, 571 (1996).

[8] M. Heusler, Black Hole Uniqueness Theorems (Cambridge University Press, England, 1996)

[9] M. S. Volkov and D. V. Gal'tson, Phys. Rep. 319, 1 (1999).

[10] D. Garfinkle, G. T. Horowitz and A. Strominger, Phys. Rev. D 43, 3140 (1991), 45, 3888(E) (1992).

[11] G. W. Gibbons Nucl. Phys. B 207, 337 (1982), G. W. Gibbons and K. Maeda, Nucl. Phys. B 298, 741 (1988).

[12] P. Kanti, N. E. Mavromatos, J. Rizos, K. Tamvakis and E. Winstanley, Phys. Rev. D 54, 5049, (1996).

[13] G. T. Horowitz hep-th/9210119.

[14] M. Rakhmanov Phys. Rev. D 50, 5155 (1994). 